\title{
Optimal Adsorption Site of Periodical Metal Surface Based on Global
}

\section{Search of Genetic Algorithm}

\author{
Jingjing Zhang ${ }^{1, \text { a }}$ \\ ${ }^{1}$ Department of Chemical Engineering and Technology, China University of Petroleum-Beijing, \\ Beijing, China, 102249 \\ a email
}

Keywords: Genetic Algorithm, Global Search, Periodical Metal Surface, Optimal Adsorption Site

\begin{abstract}
When using the chemical calculation method to study the adsorption, we should firstly find the preferential adsorption site of the catalyst surface. But at present, we often place the reactants in the typical adsorption sites, such as bridge position, vacancy and vertex position, to find the minimum energy point of the system according to the DFT structure optimization. The lack of study on atypical adsorption sites leads to the lack of other preferential adsorption sites. Therefore, we need to explore the potential adsorption sites on the periodic metal surfaces using global search method. The calculation operation of this paper involves the single point energy and structure optimization. The single point energy measures the size of the total energy of the system. The low the total energy of the system, the more stable adsorption is. The structural optimization is to change the structure to the most stable point by adjusting the coordinates of the atoms. In other words, the structural optimization is to find the most stable geometry. Structural optimization is used to calculate the configuration, which is the lowest energy structure and corresponds to the position of the optimal adsorption site. In addition, this paper also introduces the orbital analysis to verify the molecular structure of thiophene. We use the HOMO-LOMO Channel analysis to confirm whether there is $\pi$ conjugate in thiophene. Conjugate is a criterion for judging whether thiophene molecules are dissociated or adsorbed.
\end{abstract}

\section{Method}

We choose the super unit cell model to study the adsorption of thiophene molecules on Ni (100) surface. The thiophene and the two layer of the metal top are allowed to relax.

Table 1 Methods and parameters in calculating energy

\begin{tabular}{cc}
\hline Theory & DFT \\
\hline Software & Materials Studio 8.0 \\
Module & Dmol3 \\
Functional & GGA/PBE \\
Basis Set & DNP \\
Density mixing charge & 0.03 \\
SCF tolerance & $1.0 \times 10^{-3}$ \\
Smearing & $0.02 \mathrm{Ha}$ \\
\hline
\end{tabular}


Table 2 Methods and parameters in calculating geometry optimization

\begin{tabular}{|c|c|}
\hline Theory & DFT \\
\hline Software & Materials Studio 8.0 \\
\hline Module & $\mathrm{Dmol}^{3}$ \\
\hline Functional & GGA/PBE \\
\hline Basis Set & DNP \\
\hline Density mixing charge & 0.03 \\
\hline SCF tolerance & $1.0 \times 10^{-3}$ \\
\hline Smearing & $0.02 \mathrm{Ha}$ \\
\hline Convergence tolerance of energy & $2 \times 10^{-5}$ \\
\hline Convergence tolerance of Max.force & Hatree/Å \\
\hline Convergence tolerance of & $0.005 \AA$ \\
\hline Max.displacement & \\
\hline
\end{tabular}

\section{Results}

After 50 iterations, the twelve adsorption configurations of the lowest energy in the population are in the stable state. We consider the population has lost the evolutionary, that is to say the population is convergent.

Table 3 The rank of single energy of adsorption structure and the coordinates of the sulfur atom in the thiophene

\begin{tabular}{ccccc}
\hline number & Single Point Energy(Ha) & $\mathrm{X}(\AA)$ & $\mathrm{Y}(\AA)$ & $\mathrm{Z}(\AA)$ \\
\hline 1 & -11503.347 & 4.2824 & 2.8519 & 7.2833 \\
2 & -11503.347 & 6.4087 & 5.2966 & 7.1240 \\
3 & -11503.345 & 4.6802 & 2.9578 & 7.3379 \\
4 & -11503.344 & 6.4014 & 4.9455 & 7.0582 \\
5 & -11503.343 & 4.1755 & 2.8202 & 7.3130 \\
6 & -11503.342 & 6.4089 & 5.2984 & 7.1259 \\
7 & -11503.342 & 4.3019 & 2.7640 & 7.2154 \\
8 & -11503.342 & 1.6642 & 2.7526 & 7.2251 \\
9 & -11503.342 & 4.7666 & 2.0180 & 7.5835 \\
10 & -11503.339 & 2.1433 & 3.3708 & 7.3136 \\
11 & -11503.339 & 4.2731 & 2.8527 & 7.2816 \\
12 & -11503.339 & 4.1641 & 2.8206 & 7.3132 \\
\hline
\end{tabular}



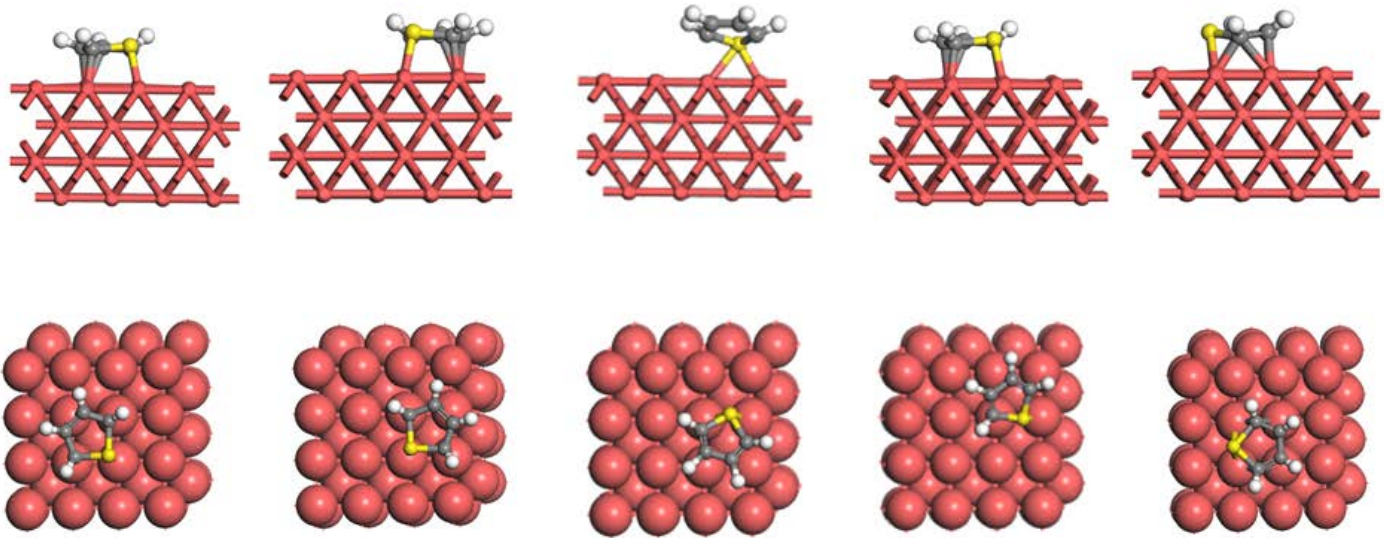

Figure 1 Front view and vertical view of adsorption structure

Table 4 The rank of single energy of adsorption structure and the coordinates ( $\AA$ ) of the sulfur atom in the thiophene

\begin{tabular}{ccccccc}
\hline & $\mathrm{X}(\AA)$ & $\mathrm{Y}(\AA)$ & $\mathrm{Z}(\AA)$ & $\mathrm{E}_{\mathrm{ad}}(\mathrm{eV})$ & $\mathrm{d}(\mathrm{S}-\mathrm{Ni}) / \AA$ & $\mathrm{d}(\mathrm{C}-\mathrm{S}) / \AA$ \\
\hline 1 & 4.554579000 & 2.907568000 & 7.460874000 & -3.462023496 & 2.161 & 1.845 \\
\hline 7 & 4.555998586 & 2.918952850 & 7.458956847 & -3.461952745 & 2.160 & 1.841 \\
\hline 11 & 4.561080000 & 2.914930000 & 7.461874000 & -3.461443883 & 2.160 & 1.842 \\
\hline 12 & 4.558490552 & 2.921046173 & 7.456357887 & -3.461288790 & 2.160 & 1.847 \\
\hline 3 & 5.414490645 & 2.909085388 & 7.459262527 & -3.461280612 & 2.164 & 1.846 \\
\hline 9 & 5.406217757 & 2.913769893 & 7.460485246 & -3.460907810 & 2.164 & 1.845 \\
\hline 2 & 7.057716244 & 5.405918923 & 7.465070895 & -3.459962140 & 2.161 & 1.846 \\
\hline 4 & 7.053928836 & 5.415587360 & 7.463389430 & -3.459710489 & 2.160 & 1.844 \\
\hline 6 & 6.463459653 & 5.261291601 & 7.334681452 & -3.430851992 & $2.477,2.208$ & 1.841 \\
\hline 10 & 2.257416732 & 3.709267990 & 7.279651831 & -3.421693540 & $2.265,2.291$ & 1.879 \\
\hline 8* & 1.257586752 & 2.976564533 & 7.183961353 & -3.490310202 & $2.492,2.304,2.302,2$. & $2.968,1.923$ \\
\hline
\end{tabular}



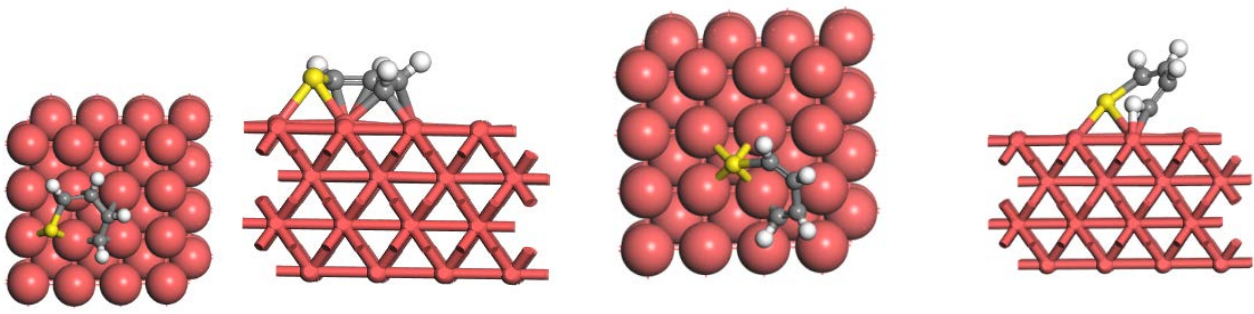

Figure 2 Front view and vertical view of adsorption structure 5 and 7

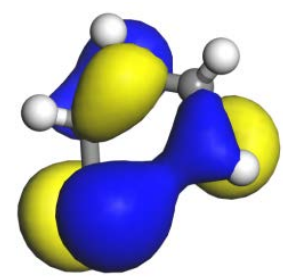

Figure 3 HOMO orbital analysis of thiophene in configuration5

The C-S bond in the adsorption configuration lengthens from $1.728 \AA$ to $1.845 \AA$ after the DFT structure optimization. The C-S bonds in all the configurations have increased and the growth rate ranges from $6 \%$ to $9 \%$; the $\mathrm{C} 2-\mathrm{C} 3$ bonds have increased by $6 \% \sim 7 \%$; the $\mathrm{C} 3-\mathrm{C} 4$ bonds have decreased $0.2 \%$ averagely; the Ni-S bonds have decreased from $2.268 \AA$ to $2.160 \AA$ compared to the literature situation and the average decreasing rate is about $4.7 \% \sim 5 \%$. It shows that the $\mathrm{Ni}$ atom and the $\mathrm{S}$ atom have bonding tendency after the reaction of thiophene and $\mathrm{Ni}(100)$ adsorbent. The carbon atom in the middle of thiophene is more close to the Ni surface. And all the $\mathrm{C}-\mathrm{H}$ bonds are tilted up, far away from the Ni (100) plane.

\section{Conclusion}

This paper combines the genetic algorithm with the adsorption system successfully, adopts the genetic algorithm and DFT method and finds the adsorption sites of periodical metal surface successfully. We use the genetic algorithm and DFT single point energy to search the existing adsorption sites on the surface of $\mathrm{Ni}$ (100). Most of the adsorption sites concentrate in the region of $(3.5,4.5)$ and $(2,4)$. Only a few adsorption sites are discrete. The distribution of relatively low energy configurations of thiophene is also retained. The change situation of C-S and S-Ni are also close to the data obtained by H Orita, which are respectively $1.845 \AA$ and $2.160 \AA$. A small number of the configurations of the discrete distribution, optimized by the DFT structure, have occurred dissociation adsorption. Although the undissociated configuration doesn't have the lowest energy, its energy is close to the lowest energy configuration, which is $-3.460 \mathrm{eV}$.

The dissociation adsorption configuration after the structure optimization by DFT, the C-S bond is broken and the thiophene ring opened. Other bonds have lengthened correspondingly. The bond of C3-C4 lengthens from $1.421 \AA$ to $1.459 \AA$. After the HOMO orbital analysis, the conjugation of $\pi$ does not exist and there is only a single bond.

The C-S bond in the adsorption configuration lengthens from $1.728 \AA$ to $1.845 \AA$ after the DFT structure optimization. The C-S bonds in all the configurations have increased and the growth rate ranges from $6 \%$ to $9 \%$; the $\mathrm{C} 2-\mathrm{C} 3$ bonds have increased by $6 \% \sim 7 \%$; the $\mathrm{C} 3-\mathrm{C} 4$ bonds have decreased $0.2 \%$ averagely; the Ni-S bonds have decreased from $2.268 \AA$ to $2.160 \AA$ compared to the literature situation and the average decreasing rate is about $4.7 \% \sim 5 \%$. It shows that the $\mathrm{Ni}$ atom and the $\mathrm{S}$ atom have bonding tendency after the reaction of thiophene and $\mathrm{Ni}(100)$ adsorbent. After the 
analysis of HOMO and LOMO orbit configuration, the conjugation of $\pi$ bond in thiophene still exist. The configuration after adsorption is the configuration of molecular adsorption.

\section{References}

[1] Wei Jun, Journal of Inner Mongolia Normal University (Natural Science Edition), Vol. 44(2015) No 3, p.372-379

[2] Li Chen, Chen Guanghua, Stoian Razvan, Acta Optica Sinica, Vol. 36(2016) No 5, p.1-7

[3] Zhou Peng, Xu Ke, Liu Shunhua, Journal of Mechanical Engineering, Vol. 51(2015) No 6, p.98-103

[4] You Wanna, China High-Tech Enterprises, Vol. 23(2016) No 1, p.77-78

\section{The implication of Zeng Ziyi’s ISTP}

The thesis named Research on Protection and Industrialization Development of Qiang Embroidery Culture in Globalization Context written by Zeng Ziyi who works in Art College of Southwest University for Nationalities has been published in Advances in Social Science, Education and Humanities Research (ETMHS 2016,ISSN:2352-5398) Vol.50 Part C. It is the Key Program of Humanities and Social Science of Sichuan Educational Committee in 2015 (Grant No. 15SA0212). It also is the Program of Postgraduate Degree Construction of Southwest University for Nationalities in 2015 (Grant No. 2016XWD-SO504). 